\title{
Experience Differentiation Strategy (2): Focus on Embodied Cognition and ACT Module
}

\author{
Shin'ya Nagasawa ${ }^{1}$, Shinich Otsu ${ }^{1,2}$ \\ ${ }^{1}$ Graduate School of Commerce, Waseda University, Tokyo, Japan \\ ${ }^{2}$ IBM Japan, Ltd., Tokyo, Japan
}

Email address:

nagasawa@waseda.jp (S. Nagasawa)

\section{To cite this article:}

Shin'ya Nagasawa, Shinich Otsu. Experience Differentiation Strategy (2): Focus on Embodied Cognition and ACT Module. Science Journal of Business and Management. Special Issue: Customer Experience Management/MarketingBranding.

Vol. 3, No. 2-1, 2015, pp. 78-82. doi: 10.11648/j.sjbm.s.2015030201.21

\begin{abstract}
In a mature market like recent Japanese economy, "experiential marketing" has received attention as "differentiation strategy." In this paper, we focus on behavior experiences (ACT Module of SEMs) in terms of "Embodied cognition." Behavior experiences (ACT Module) are consumer's behaviors and physiological /psychological effects occurs by consumer's behaviors. The results shows that there are two points for leading to Behavior experiences (ACT Module), 1) design products that has factors leading to consumers behaviors, 2) design situations that has factors leading to consumers behaviors.
\end{abstract}

Keywords: Customer Experience, Consumer Experience, Differentiation Strategy, Embodied Cognition, Behavioral Experience

\section{Background and Purpose}

\subsection{Customer Experience Strategy}

The customer experience strategy aims to establish a unique position for products and services in the minds of consumers by focusing on that which is experienced when a product or service is consumed or utilized and then differentiating those experiences.In their previous thesis "Experience Differentiation Strategy: Concepts of Consumer Experiences," the authors presented the concept of consumer experience as a target for such differentiation as described below.

Consumer experience refers to consumer behavior as well as physiological and psychological effects in various situations that surround consumers and that they directly experience during the process of obtaining, consuming, and disposing of products and services.

Consumer experience is comprised of three characteristics: (1) the process of consumption (obtaining, consuming and disposing of products and services), (2) subjectivity (in the situations that surround consumers and are experienced directly); and (3) both external and internal behavior (consumer behavior and the psychological and physiological effects on consumers). Customer experience strategy aims to establish a unique position for products and services in the minds of consumers and gain competitive edge by differentiating consumer experiences from those of competitors.

\subsection{Strategic Experiential Modules (SEMs) by Schmitt}

Based on notions of cognitive science, Schmitt pointed out that there are five types of experiences (Strategic Experiential Modules or SEMs [1]) as classified below in Table 1.

The purpose of the SEMs concepts is to classify experiences so that they can easily be used as components in constituting the goals and strategies of marketing activities. SEMs are being researched by the Nagasawa laboratory at Waseda Business School on a continuous basis [2]-[7].

\subsection{Purpose of This Article Differentiation Through Behavior Experiences}

Based on the background described above, this article focuses on ACT (behavior experiences), one of the five SEMs put forward by Schmitt, as a specific customer experience. Together with RELATE (relational experiences), it is said that ACT is the most difficult to understand of the SEMs, and some critics wonder whether it is possible to realistically integrate 
Table 1. Strategic Experiential Modules (SEMs).

\begin{tabular}{|c|c|c|}
\hline Module & Keywords & Examples \\
\hline SENSE (Sensory experiences) & Senses & I felt cold when I touched the ice. \\
\hline FEEL (Emotional experiences) & Feelings & When I watched the movie, I thought that it was heartwarming. \\
\hline THINK (Cognitive experiences) & Curiosity & $\begin{array}{l}\text { After hearing that the latest mobile phone had been launched, I went to a consumer } \\
\text { electronics shop to check it out. }\end{array}$ \\
\hline ACT (Behavioral experiences) & Action & I started jogging, and I felt good. \\
\hline RELATE (Relational experience) & Feelings of being connected & I joined a community on SNS. \\
\hline
\end{tabular}

Source: Authors' elaboration based on Schmitt [1].

ACT into products and services [5]. The objective of this article is to build methodologies to create and differentiate ACT (behavior experiences).

\section{What is ACT (Behavioral Experiences)}

\subsection{Act as Defined by Schmitt}

Schmitt defined ACT as "physical experiences, lifestyles, and experiences that emphasize interactions with other people." In his definition of ACT, while the concept of physical experiences is difficult to understand, "interactions with other people" are easily confused with RELATE because they do not at first appear to fit in with ACT. The difficult concept of ACT leads to poor carryover into the field of marketing.

According to Schmitt, ACT is derived from the notion of embodied cognition [1]. In this chapter, the authors reanalyze the concept of ACT by delving deeper into the idea of embodied cognition.

\subsection{Embodied Cognition}

The idea of embodied cognition holds that cognition, including perception, is related to physical movements and that it interacts with its environment through such movements [8]. Embodied cognition is a new notion that was put forward in opposition to the information processing model (input -> processing $->$ output) that is based on conventional cognitive science.

In the information processing model, the idea is that people perceive environmental information, make a judgment and determine their intentions, and then produce work on the environment through locomotion. Therefore, movements are not made until information processing is complete, and often what results greatly differs from the decisions people make and the actions they take in an instant. In order to solve this problem, researchers put forward the idea of embodied cognition in which they introduce James J. Gibson's ideas in ecological psychology and considered the body and its environment as a whole system in which the two interact [8].

\subsection{Ecological Psychology}

As described earlier, the concept of embodied cognition draws from ideas from ecological psychology. The notion of ecological psychology was put forward by James J. Gibson, who introduced principles of ecology, which emphasize interactions between animals and their environment, into psychology [8]. One characteristic of ecological psychology is that animals and their environment are considered a larger system called an "ecosystem" in which the two interact [9].

In ecological psychology, there is also the concept of affordances. "The affordances of the environment are what it offers the animal, what it provides or furnishes, either for good or ill" (Gibson, 1985, p. 137 [9])." The term was coined by Gibson based on the verb "afford," which means to "be in a position to do something" or "provide." Its slightly easier definition is opportunities for action animals receive from the environment in which they live [9]. Animals search for affordances, and choose them to take action. It can be said, for instance, that chairs afford opportunities for sitting or that they provide affordances for sitting.

As described above, ecological psychology sees physical movements as determined by interactions between the body and its environment and refers to the environmental characteristic of such interactions as "affordances."

\subsection{What is ACT (Behavior Experiences)}

Schmitt adapted the notion of embodied cognition to his concept of ACT (behavior experiences). In this article, the authors reanalyze the concept of ACT based on the notion of embodied cognition, and redefine it as follows:

Consumer behavior and physiological and psychological activities that arise as a result of such behavior during activities of consumption

Based on the idea of embodied cognition that people recognize the environment through their physical movements, it can be said that ACT consists of both consumer behavior and physiological and psychological activity that arises as a result of such behavior. For example, the physical movements of running, sitting, and holding or the acts of studying, driving a car, and going to a park, and the resultant senses and emotions of feeling good, refreshed, and happy are all included in ACT. Therefore, this article redefines ACT.

\section{Creation of ACT}

\subsection{Interactions between $A C T$ and products}

Based on the notion of embodied cognition and principles of ecological psychology, ACT is generated through interactions with the environment. In the context of consumer experiences such as obtaining, consuming, and disposing of products and services, the most influential environment is products and services. In other words, interactions between products and services and consumers generate ACT on the 
part of consumers. Physical movements and actions are generated depending on how products and services are consumed, and in the process of their consumption, emotions such as feeling good and happy are generated.

The pleasant feeling one has when closing the door of a car and the comfortable feeling one experiences when opening a folding mobile phone are examples of product-related movements and actions and resultant feelings of consumption, and these represent ACT. And these movements and feelings of consumption are greatly affected by product design. Nintendo's home computer game machine "Wii" offers new game operations such as shaking, turning, and aiming through use of the Wii remote control as part of its product design.
Norman called incorporating the pleasure and utility of product consumption with product design "behavioral design," and pointed out its importance [10]. He also indicated affordance as an important element of a product that evokes and determines feelings on their consumption [11]. In the field of product design, Fukazawa also referred to affordances and then pointed out the importance of designs that took human behavior into consideration by calling such interactions "designs that respond instantaneously to behaviors" [12].

As mentioned above, both Norman and Fukazawa pointed out that in order to create ACT on the part of consumers, it is important to incorporate affordances into product designs (Figure 1).

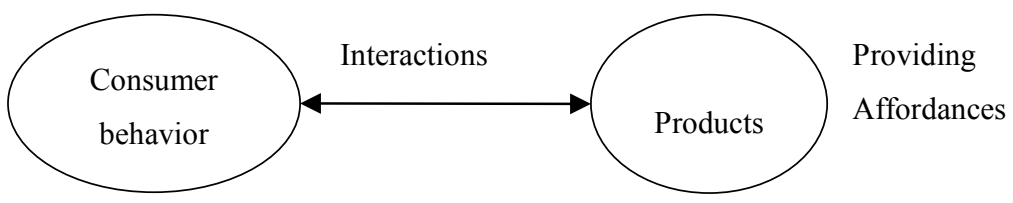

Figure 1. Interactions between Consumer Behavior and Products.

Source: Authors' elaboration.

\subsection{Case Study 1: Inax Satis}

This article reanalyzes INAX's SATIS as an example of the interaction between product and consumer behavior. Nagasawa et al. originally chose SATIS, INAX's toilet for small spaces, as an example of creation of customer experiences [2].

The SATIS tankless toilet was launched by INAX in 2001. By eliminating its tank, SATIS reduced its depth by $15 \mathrm{~cm}$ by lessening the previous $80 \mathrm{~cm}$ to $65 \mathrm{~cm}$, thus achieving space-saving and making the bathroom feel larger. The conventional toilet with a tank had a faucet in the tank to enable users to wash their hands. By installing a separate washstand with a mirror in the bathroom, the tankless toilet separated flushing from running water to wash hands. As a result, one can now use the bathroom only to wash one's hands or freshen up one's makeup which comes in handy for activities such as when guests are invited to a home party and wish to use the bathroom as a place to be alone or fix one's attire.

As described above, SATIS offered new way of acting to consumers. In other words, consumers experience new behavior as they use SATIS. This is really an example of the creation of an ACT (behavior experience) by a product.

\subsection{Act and Interactions with Situations}

The previous section examined interactions between products and services with ACT. Based on the idea of embodied cognition, which holds that ACT is generated through interactions with the environment, products and services are not the only environment that affects consumers.
There are also environments other than products and services. In this section, these other environments are called "situations." It is assumed that such situations affect consumer behavior (Figure 2).

Examples of situations include products and services other than those which are being consumed directly; consumers other than oneself; the circumstances that have existed, and the knowledge that has been acquired up to the time of purchase; and the timing of obtaining, consuming, and disposing. All factors related to consumption beyond products and services fall into this category. It can be said that all factors other than products and services that are recognized by consumers are included in the concept of situations. Situations are one of the three characteristics that comprise the concept of experiences presented in Chapter 1, and they are also an important factor in the entire experience, not to mention ACT.

More specifically, consider the situation of being hungry that leads to the act of eating, or being invited by a friend to watch a movie together that can lead to the act of doing so. The differences in the situation between having a meal alone and doing so with somebody else sometimes bring different feelings and emotions to the act of eating. Crowded and almost empty trains evoke different feelings when riding them. Furthermore, depending on when one takes an almost empty train - that is, during the daytime or nighttime - situational differences may bring different feelings to the act of taking a train.

As described above, situations also affect the behaviors as well as feelings and emotions of consumers when they consume products and services. 


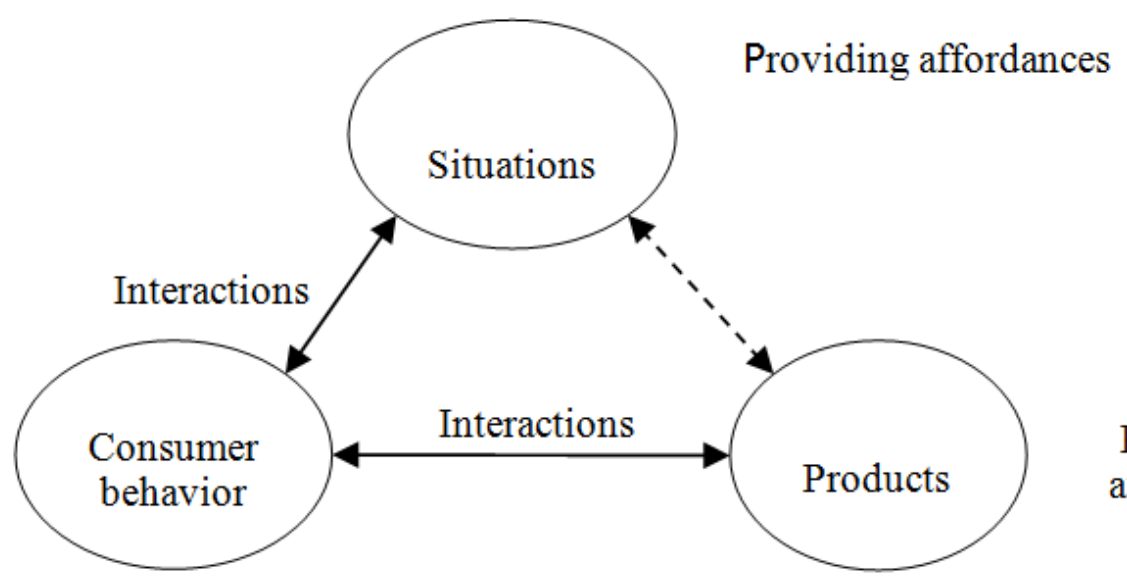

Providing affordances

Figure 2. Consumer Behavior and Situation Source: Authors' elaboration.

\subsection{Case Study 2: Albirex Niigata}

This section reexamines Albirex Niigata, a J. League Division 1 football club, an example of creation of customer experience that Nagasawa et al. originally used (Nagasawa, 2005), from the viewpoint of situations and ACT.

The ACT provided by Albirex Niigata is the festive experience that comes from supporting a football club with other supporters in a stadium that accommodates 40,000 spectators. Both supporting the football club as part of the 40,000 spectators and becoming excited when doing so make this experience special. The important point of ACT creation lies in the fact that with 40,000 spectators, the stadium is packed to its capacity. In this case, the situation, and not the product itself is important.

Albirex Niigata had the intention of mobilizing 40,000 people because there was a stadium that accommodated 40,000 people, and attempted to create a place of enthusiasm for all 40,000 people. Specifically, in order to have local residents understand the plans for creating such a space, the football club distributed a large number of free admission tickets. Of course, the distribution of free tickets would not always lead local residents to actually come to the stadium. It was even rumored that other J. League teams failed when distributing free tickets. But Albirex Niigata racked its brain and made efforts in various ways that included using town associations to distribute free tickets to those who really wanted to go to the stadium and obtaining the cooperation of local boards of education. As a result, the football club succeeded in creating a situation in which the stadium was filled with 40,000 spectators from the very beginning. And it created not only the experience of watching the game and supporting the team, but also the special behavior experience of watching the game at a stadium packed with 40,000 other spectators while supporting the team enthusiastically and feeling unified with other spectators. This made watching the football game an attractive experience.

As described above, Albirex Niigata is a good example of ACT derived not from the product itself (the football game) but from the situation.

\section{Proposals to Integrate ACT into Products and Services}

The preceding sections indicate that the two most important points for creating ACT are (1) design factors that derive behavior experiences for products and services and (2) design factors that derive behavior experiences for situations in which products and services are consumed.

Norman and Fukazawa have heretofore proposed that factors for behavior experiences should be designed directly for products and services. This is one way of integrating ACT into products and services. There has not been much past mention of environmental factors (or situations) other than products and services, however. In order to create and differentiate ACT, it is therefore desirable to integrate ACT-deriving factors into situations of consumption as well as into products and services. In the case of Albirex Niigata, the situational factor that differentiated its ACT was a "stadium filled with 40,000 spectators."

Integrating ACT-deriving factors into situations means creating special situations. Experiences in special situations become special experiences. Examples of special situations may include illogical or unusual situations.

A possible illogical situation could be one of low accessibility or high inaccessibility. The conventional marketing theory strives to provide greater accessibility to consumers. This means making products as accessible as possible mainly through wider distribution channels. But one possible way of creating an illogical situation is to intentionally put products and services in a situation that makes them highly inaccessible. Examples include restaurants that are difficult to find or Chinese noodle shops that always have a line out the door. The fact that a line is formed in front of a shop means that the shop always makes it necessary for consumers to wait, lowering its accessibility. Such a special situation with low accessibility creates a special experience. The situation of taking time and being inconvenient sometimes creates valuable, special experiences though this visibly contradicts conventional wisdom. 
Unusual situations refer to ones that cannot be experienced in daily life. For example, a "stadium filled with 40,000 spectators" falls into this category. Such situations mean that one is placed in an environment different from daily life. To Japanese people who live in a country where Japanese is spoken in daily life, the inability to make oneself understood in Japanese when traveling overseas is an unusual situation. Such a situation makes it necessary to communicate in languages other than Japanese, bringing about a behavior experience of communicating in non-Japanese languages. Another example of an unusual situation is Hoshinoya Kyoto, a luxury Japanese inn in Kyoto, which can be reached only by boat from a port in Arashiyama because it is inaccessible by car. The Japanese inn creates an illogical situation that lowers its accessibility yet at the same time creates the unusual experience of arriving by ferry.

It can be said that these special situations derive special behavior experiences. The important point in differentiation through ACT lies in how marketers design factors that spark behavior experiences for products and services, while making situations at the time of consumption special to beget behavior experiences.

\section{Conclusion}

This article defined ACT and described how it is created from the viewpoint of embodied cognition, ecological psychology, and affordance.

ACT refers to consumer behavior as well as physiological and psychological activities that arise as a result of such behavior at the time of consumption. The two important points to create ACT are the (1) design factors that derive behavior experiences for products and services and (2) design factors that derive behavior experiences for situations in which products and services are consumed. We also showed that one possible way of invoking special behavior experiences is to create illogical and unusual situations.

These ways of thinking will help marketers to understand consumers' behavior experiences and use ACT for marketing from the perspective of the consumer experience. In this article, the authors only propose specific ways of creating situations, more specifically illogical and unusual situations, for products and services to differentiate ACT, but do not examine them fully. This is an issue to be addressed in the future.

\section{References}

[1] Schmitt, Bernd H. (1999), Experiential Marketing: How to Get Customers to Sense, Feel, Think, Act, and Relate to Your Company and Brands, Free Press, Detroit.

[2] Nagasawa, S. ed., Waseda Business School Nagasawa Laboratory (2005), Creation of Customer Experiences That Bring Hit Products: Manufacturing That Excites the Kansei of Consumers, JUSE Press, Tokyo (in Japanese).

[3] Nagasawa, S. ed., Waseda Business School Nagasawa Laboratory (2006), Creation of Customer Experiences by Companies with Long-established Brands: Design Management to Meet Customers, Doyukan, Tokyo (in Japanese).

[4] Nagasawa, S. ed., Waseda University Business School Nagasawa Laboratory (2007), Manufacturing for Customer Experiences: Brand Value and Creation of Situations to Produce Hit Products, JUSE Press, Tokyo (in Japanese).

[5] Nagasawa, S. and T. Someya (2007), The Tradition of the Long-established Brand Toraya and its Innovation: Creation of Customer Experiences and Technology Management, Koyo Shobo, Tokyo (in Japanese).

[6] Nagasawa, S. ed., Waseda Business School Nagasawa Laboratory (2009), Premium Brand Strategy for Local and Traditional Industries: Technology Management That Brings Customer Experiences, Doyukan, Tokyo (in Japanese).

[7] Nagasawa, S. and M. Ishikawa (2010), Kyo-yuzen Chiso: Innovation in the 450-year-old Brand, Doyukan, Tokyo (in Japanese).

[8] Higuchi, T. and S. Morioka (2008), Body Mechanics, Miwa Shoten, Tokyo (in Japanese).

[9] Sasaki, M. and H. Mishima (2001), Ideas of Affordance, University of Tokyo Press, Tokyo (in Japanese).

[10] Norman, D.A. (2004), Emotional Design: Why We Love (or Hate) Everyday Things, Basic Books, New York.

[11] Norman, Donald A. (1990), Design of Everyday Things, Doubleday Business, New York.

[12] Fukazawa, N., M. Sasaki, and T. Goto (2004), The Ecology of Designs, Tokyo Shoseki, Tokyo (in Japanese). 